\title{
ACTION POTENTIAL VELOCITY DETECTION USING A PENETRATING MICROPROBE
}

\author{
Michael P. Hughes*, Danny J. Banks and David J. Ewins \\ Centre for Biomedical Engineering, School of Engineering, \\ University of Surrey, Guildford, Surrey GU2 7XH, UK \\ *Corresponding author. \\ Tel: +44 (1483) 686775 \\ Fax: +44 (1483) 689395; \\ Email: m.hughes@surrey.ac.uk
}

\begin{abstract}
The use of a single implantable microprobe to detect multiple, bi-directional action potential velocities is demonstrated. By using multiple electrode sites along the direction of propagation to record the same action potentials, recorded action potentials can yield the velocities of a number of neurons in both afferent and efferent directions.
\end{abstract}




\section{INTRODUCTION}

For a number of years, researchers have devised implantable electrode arrays for neurophysiological measurement (Heiduschka and Thanos, 1998). A number of interface designs have been proposed, including the penetrating probes such as those developed by Wise and co-workers (e.g. Drake et al., 1988). Such probes usually consist of one or more needle structures that are inserted into the nerve, and which carry electrode sites that sample electrical conditions within the nerve. Probes are generally micromachined from silicon using processes developed by the semiconductor industry.

We propose that penetrating probes carrying a number of electrodes along the width of the shank can be used to determine the velocity of neural signals (or Action Potentials, AP) by timing the pulse peak from one electrode to the next. In this paper we demonstrate that it is possible to measure the velocity of many APs simultaneously, and use the velocity as a means of discriminating between them, using the wellcharacterised (e.g. Pearson et al., 1970; Gwilliam and Burrows, 1980), unmyelinated locust nerve cord as a model. Since important nerve functions associated with, for example, touch, pressure and joint position are carried on larger, faster neurons we might wish to identify these APs from those carried on slower neurons.

\section{METHODS}

The microprobes used consisted of shanks of flat cross-section, typically $6 \mu \mathrm{m}$ thick and $100 \mu \mathrm{m}$ wide, and extended $1-2 \mathrm{~mm}$ in length. The tip of a typical device is shown in figure 1. The experiments presented here specifically used a microprobe bearing two arrays of electrodes running perpendicular to the principal shank of the probe, with the two arrays stacked as shown in the micrograph in figure 1 . The lower 
three electrodes measured $12 \times 12 \mu \mathrm{m}$, whilst the upper sites measured $4 \times 4 \mu \mathrm{m}$. Interelectrode gaps along the axis of conduction were $36 \mu \mathrm{m}$. These sites therefore form a rectangular array capable of detecting signals in the directions parallel and orthogonal to the main axis of the shank.

Microprobes were fabricated using BESOI (Bonded, Etched-back Silicon On Insulator) wafers. The fabrication process, developed from work by Ensell et al. (2000), is summarised here. The probes consisted of two silicon wafers, one $525 \mu \mathrm{m}$-thick and the other etched back to $6 \mu \mathrm{m}$ thick, with a $1 \mu \mathrm{m}$-thick layer of oxide separating them. This allowed probe shapes to be defined without requiring high levels of boron doping, with the advantage that such probes may be used as the basis for integrated on-chip electronics at a later date. A $600 \mathrm{~nm}$ layer of silicon dioxide and a $200 \mathrm{~nm}$ layer of silicon nitride were deposited, and were patterned by reactive-ion etching and etched in $\mathrm{KOH}$ in order to form the probe shape. Oxide and nitride films were then removed by wet etching. The wafers were then covered in a $1 \mu \mathrm{m}$ thick oxide layer to isolate the silicon from the metallization, followed by a 160nm layer of LPVCD nitride. Electrode patterns comprised a 40nm seed layer of chrome anchoring a 300nm layer of gold. The metal was patterned using ion beam milling, and covered with a 500nm thick passivation layer of silicon nitride. Via holes to the recording sites and bonding pads were reactive-ion etched, and the probe structure was released from its frame with a final etching of the silicon superstructure.

The probe was attached directly to a five-channel headstage amplifier with channel voltage gain of 100 . Signals were fed from the headstage amplifier into a second stage, 5-channel custom amplifier unit that further amplified the signal, as well as providing initial signal conditioning (specifically a $100 \mathrm{~Hz}$ notch filter for removing 
mains interference). The gain of the unit could be varied between $x 5$ and $x 75$. The conditioned signals were then fed into an ADC card (model AT-MIO-16F-5, National Instruments) mounted on a PC, which sampled at a maximum 40kHz/channel and also provided a gain of $\mathrm{x} 0.5$ to $\mathrm{x} 100$. The total gain provided by the instrumentation could therefore be varied between 250 and 750,000 over a bandwidth of $100 \mathrm{~Hz}-10 \mathrm{kHz}$. The gain of the system was 10,000 . The instrumentation was managed using LabVIEW (National Instruments) software. Recorded signals were saved as a series of five arrays, each relating to the input from a specific channel. This data was then loaded into MATLAB $^{\circledR}$ (The Mathworks, Inc) for postprocessing and analysis. Experimental data were recorded from the nerve trunk supplying the metathoracic leg of the adult migrating locust. Locusts were sacrificed by decapitation, pinned down on wax and immersed in locust saline as described by Hoyle (1953), and dissected to expose the nervous system. The probe was inserted into the nerve and advanced in $10 \mu \mathrm{m}$ steps using a micromanipulator. The probe was inserted into the nerve with its long axis projecting through the nerve, and the shortest axis across the nerve, so that the nerve fibers were only displaced by the $6 \mu \mathrm{m}$ edge of the probe. Insertion was performed manually by examination using a stereomicroscope (Meiji EMZ, Tokyo). All potentials were measured relative to a gold wire electrode immersed in the saline solution some distance from the nerve. Recordings were taken at various positions through the nerve, with several half-second samples being taken over a period of several minutes. The neural activity was not observed to diminish significantly during the experiments, which typically lasted 10-15 minutes. The data presented were recorded in three locusts, each using a different probe. Touching the locust leg elicited greater neural activity, but 
disturbed the positions of probe and nerve; the recordings used in this analysis were taken when no external stimulus was applied.

The velocity of AP propagation was calculated by measuring the time between significant signal peaks occurring in the record of two adjacent electrodes sites a known distance apart. An example of recorded data is shown in figure 2, where three APs were clearly observed in both channels. The number of APs seen at any time varied according to the position of the probe in the nerve. The maximum velocity that could be measured was limited by the sampling rate of the ADC card $(40 \mathrm{kHz})$. In this case the maximum speed was $1.44 \mathrm{~ms}^{-1}$. Due to the difference in size (and hence, impedance) of the electrodes, it is estimated that the amplitude of potentials on the $4 \mu \mathrm{m}$ site should be approximately $20 \%$ smaller than the same signal recorded from the $12 \mu \mathrm{m}$ site. However, this did not impede the observation of APs on electrodes of either size. Noise was observed in the recorded potentials from all electrodes; a $225 \mu$ s rolling average filter was found to remove the majority of the noise without affecting the neural data. Spectral analysis of electrode noise (Prohaska et al., 1986) was performed, which indicated that the frequency content of the noise follows a $1 / \mathrm{f}$ trend, and the majority of the noise signal was of sufficiently low frequency that it was unlikely to affect the timing of the AP spikes; the sample number of the amplitude peak will only be disturbed by high frequency noise, with the more significant low-frequency noise (evident in figure 2) changing the amplitude of the signal but not the timing.

\section{RESULTS AND DISCUSSION}

The velocities of 120 recorded APs were studied both parallel and perpendicular to the axis of the nerve using data from all four electrode sites. The distribution of the 
velocities of APs along the direction of the nerve is presented in figure 3. It can be seen that the measurements indicate that in most cases the velocity of propagation is $1.44 \mathrm{~ms}^{-1}$ or greater. Above that velocity, APs are observed to occur simultaneously on both electrodes. The distribution then tails away in both directions, with few velocities of value less than $0.3 \mathrm{~ms}^{-1}$ being observed. Assuming (as discussed earlier) that timing is unlikely to be affected by noise due to the high frequency of the peak and the $1 / \mathrm{f}$ relationship of noise power which limits thermal noise at peak frequency, the principal source of timing errors is in the timing of APs which cross the electrode array during the sample interval. In this case, the peak will appear in two subsequent sampling rounds and the timing of $0.25 \mu$ s will be too slow. In order to compensate for this it is important to interpret each velocity quoted as being a minimum, with some APs of greater velocity falling in the velocity band below. Significantly, measurements were observed in both directions, i.e. both afferent and efferent signals were detected. It is known (Cook, 1951) that the locust peripheral nervous system contains a number of spontaneously firing motor neurons. However, the direction of AP travel was strongly biased in one direction. Whilst one might speculate regarding whether the majority of APs are afferent or efferent, we have no information about this at present. Pearson et al. (1970) determined that for locust neurons with measured diameters ranging from below $2 \mu \mathrm{m}$ to greater than $10 \mu \mathrm{m}$, there exists a logarithmic relationship between neuron diameter and conduction velocity. Velocities of $0.3-3 \mathrm{~ms}^{-1}$ have been reported (Pearson et al., 1970; Gwilliam and Burrows, 1980). The distribution of neural diameters in the ventral nerve cord of the locust was measured by Cook [8], who reported that approximately $12 \%$ of neurons have diameters of $1 \mu \mathrm{m}, 12 \%$ have diameters of $2 \mu \mathrm{m}$, $12 \%$ have diameters of $3 \mu \mathrm{m}, 8 \%$ of neurons have diameter $4 \mu \mathrm{m}$, and $56 \%$ of neurons 
have diameters of $5 \mu \mathrm{m}$ or greater. According to the results of Pearson et al. (1970) neurons having these diameters have conduction velocities of approximately $0.3 \mathrm{~ms}^{-1}$, $0.5 \mathrm{~ms}^{-1}, 0.7 \mathrm{~ms}^{-1}, 1 \mathrm{~ms}^{-1}$ and $1.5 \mathrm{~ms}^{-1}$ or greater. This compares well with the recorded data in which $6 \%$ of observed APs had conduction velocity $0.36 \mathrm{~ms}^{-1}$ or less, $13 \%$ of approximately $0.5 \mathrm{~ms}^{-1}, 27 \%$ having values between 0.7 and $1.4 \mathrm{~ms}^{-1}$ and $54 \%$ having values greater than $1.4 \mathrm{~ms}^{-1}$. Such results are well within acceptable tolerances when considering the natural variations of neuron diameters between locusts and the limits of the sampling rate. In the case of signals detected in the axis perpendicular to the nerve, $48 \%$ of AP peaks occurred simultaneously (i.e. both peak on the same sample). A further $33 \%$ were within one sample of one another, with a strong bias in one direction ( $24 \%$ to $9 \%$ ). The remaining $19 \%$ were observed to be displaced by 2 samples (biased in the same direction as before, $12 \%$ to $7 \%$ ). Since in this case the AP should be travelling at $90^{\circ}$ to the longest axis of the electrodes, it is anticipated that simultaneous recording should be seen. The occasional presence of a delay of one sample may be indicative of two effects. Firstly, it may be due to noise causing a drift in the perceived peak signal timing. Alternatively, if the angle between the axis of the probe and the axis of the nerve is more or less than $90^{\circ}$, there is a non-zero distance between the electrodes along the axis of the probe. This might be due either to the probe having entered the nerve at a non-orthogonal angle, or due to any neural fibre undulation such as that described in rats by Struijk and Schnabel (2000). The proportion of the directional bias across the nerve is almost identical to that observed for action potentials along the nerve (2:1 and 1.83:1 respectively). This suggests that the most likely cause of the nonsimultaneous AP detection is the non-orthogonal angle between neurons and probe, which may in turn suggest that the likely effect of noise on recordings is low. The 
presence of tilt in the probe would also lead to an error in the perceived velocity of APs along the nerve; a tilt on $30^{\circ}$ from the vertical would result is a perceived $15 \%$ increase in AP velocity along the nerve, and a perceived AP velocity perpendicular to the nerve at twice the actual AP velocity. However, the good correlation between predicted and measured results indicates the angle of insertion is no larger than this.

\section{CONCLUSION}

Using a single microprobe, it has been shown to be possible to determine action potential velocity and direction for multiple AP sources in real time. Such a system offers many advantages over traditional single-needle recording methods, and if combined with existing methods of triangulation and signal processing, as well as improved spike detection software using interpolation or wavelets, may allow the transmission of neural data to be mapped in 3D around the local area of the electrode.

\section{ACKNOWLEDGEMENTS}

The authors wish to thank Dr. Graham Ensell at Southampton University for probe fabrication, Rutherford Laboratories for mask fabrication, and Dr. Peter Richards for valuable discussions. Probes and signal processing were funded by the EPSRC (UK), grants. GR/K16975 and GR/N65806 respectively. 


\section{REFERENCES}

Cook, P. M. (1951). Observations on giant fibres of the nervous system of locusta migratoria. Quarterly journal of Microscopical Science 92. 297-30518.

Drake, K. L., Wise, K. D., Farraye, J., Anderson, D. J. and BeMent, S. L. (1988). Performance of planar multisite microprobes in recording extracellular singleunit intracortical activity. IEEE Trans. Biomed. Eng. 35. 719-732.

Ensell, G., Banks, D. J., Richards, P. R., Balachandran, W. and Ewins, D. J. (2000). Silicon based microelectrodes for neurophysiology, micromachined from silicon-on-insulator wafers. Med. Biol. Eng. Comput. 38. 175-179.

Gwilliam, G. F. and Burrows, M. (1980). Electrical characteristics of the membrane of an identified insect motor neurone. J. Exp. Biol 86. 49-61.

Heiduschka, P. and Thanos, S. (1998). Implantable bioelectronic interfaces for lost nerve functions. Prog. Neurobiol. 55. 433-461.

Hoyle, G. (1953). Potassium ions and insect nerve muscle. J. Exp. Biol. 30. 121-135.

Pearson, K. G., Stein, R. B. and Malhotra, S. K. (1970). Properties of action potentials from insect motor nerve fibres. J. Exp. Biol. 53. 299-316.

Prohaska, O. J., Olcaytug, F., Pfundner, P. and Dragaun, H. (1986). Thin-film multiple electrode probes: possibilities and limitations IEEE Tans. Biomed. Eng. 33. 223229.

Struijk, J. J. and Schnabel, V. (2000). Influence of parameter variability on stimulus thresholds in nerve fiber models. Proc. $5^{\text {th }}$ Annual Conference of the International Functional Electrical Stimulation Society (Aalborg, Denmark) 245-248. 


\section{FIGURES}

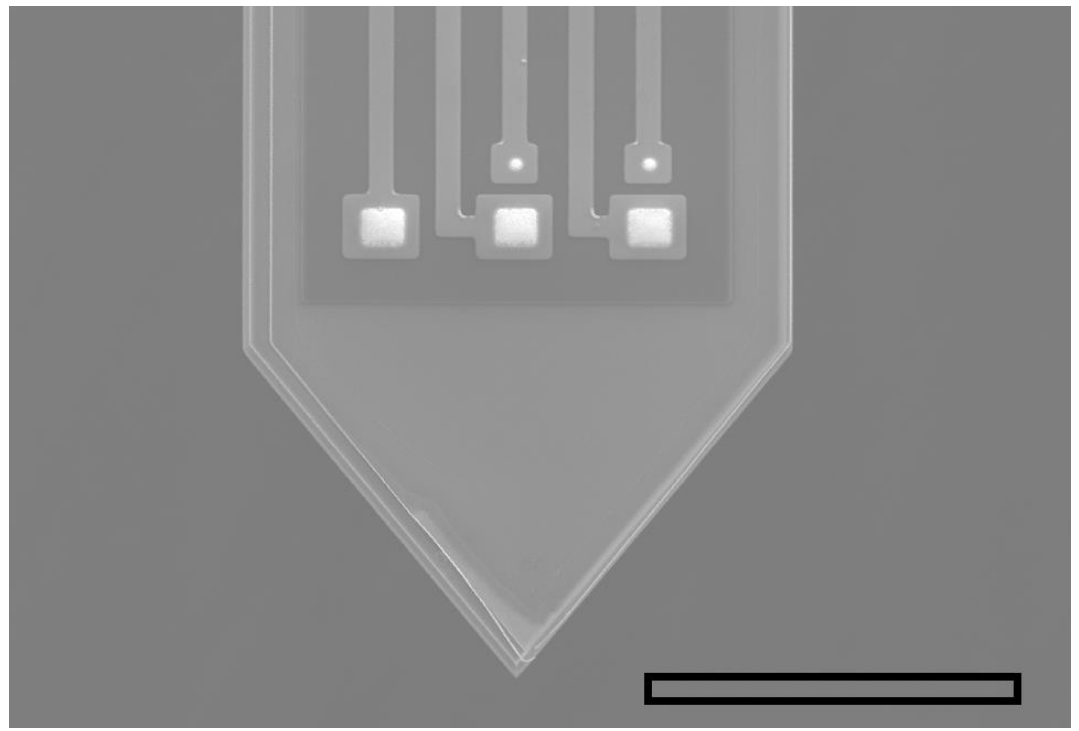

Figure 1. An electron micrograph of the tip section of a microprobe used in this study.

The electrode areas appear as white squares in the raised, lighter regions indicating the location of the gold pads and tracks. Scale bar: $100 \mu \mathrm{m}$. 


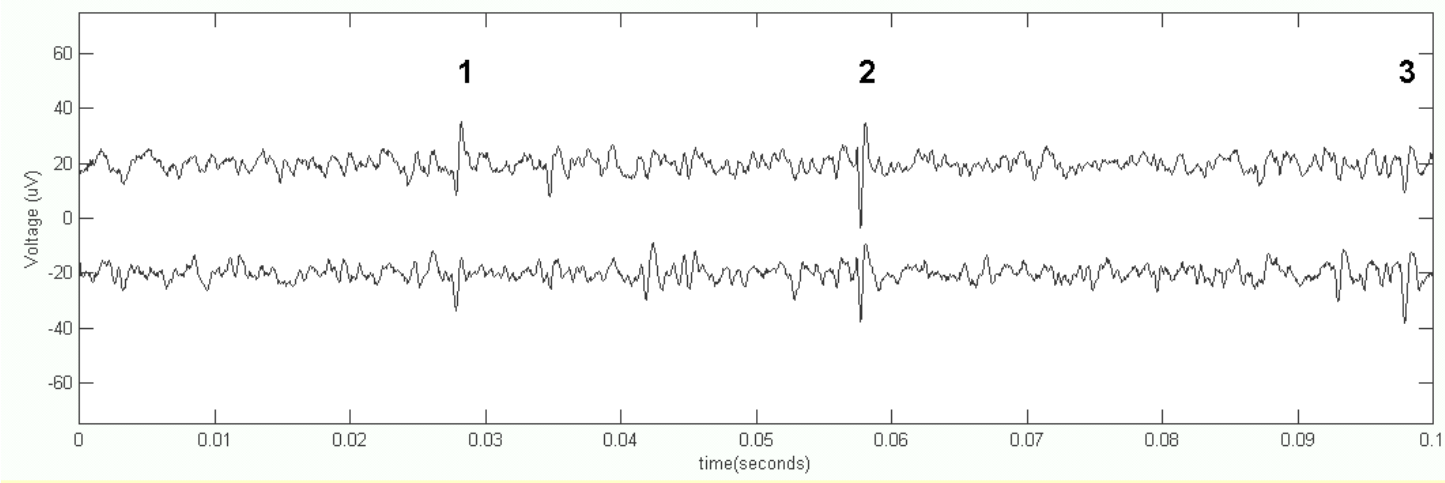

Figure 2. Recordings of $100 \mathrm{~ms}$ of neural activity using two electrodes spaced $36 \mu \mathrm{m}$ apart. The figure shows a number of action potential spikes which occur in both channels; three action potential spikes are indicated. 


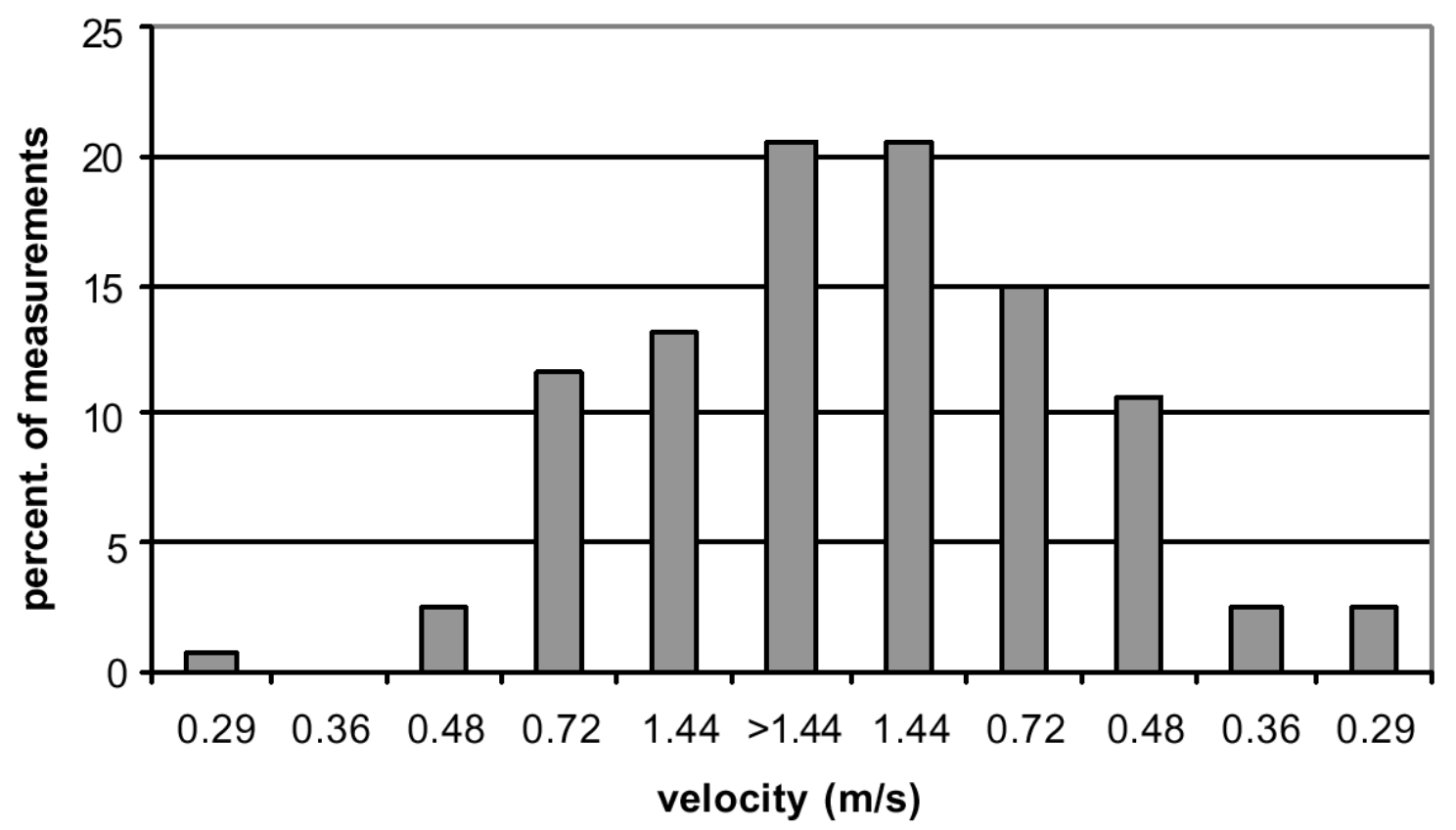

Figure 3. The distribution of propagation velocities recorded in the nerve trunk supplying the metathoracic leg of the locust. The majority of signals conduct with velocities of $1.44 \mathrm{~ms}^{-1}$ or more; the column marked "> $>1.44$ " indicates signals travelling faster than this in either direction, since spikes appeared in both channels simultaneously. 